\title{
A shallow-water theory for annular sections of Keplerian disks
}

\author{
O. M. Umurhan ${ }^{1,2,3}$ \\ 1 Astronomy Unit, School of Mathematical Sciences, Queen Mary University of London, London E1 4NS, UK \\ e-mail: mumurhan@ccsf.edu \\ 2 Department of Geophysics and Space Sciences, Tel-Aviv University, Tel-Aviv, Israel \\ 3 Astronomy Department, City College of San Francisco, San Francisco, CA 94112, USA
}

Received 3 February 2008 / Accepted 21 July 2008

\section{ABSTRACT}

\begin{abstract}
Context. We present a scaling argument that we develop into a shallow water theory of non-axisymmetric disturbances in annular sections of thin Keplerian disks.

Aims. We develop a theoretical approach to understand physically the relationship between two-dimensional vortex dynamics that is known and their three-dimensional counterparts in Keplerian disks.

Methods. Using asymptotic scaling arguments, varicose disturbances of a Keplerian disk are considered on radial and vertical scales consistent with the height of the disk while the azimuthal scales are the full $2 \pi$ angular extent of the disk. For simplicity perturbations are assumed to be homentropic according to a polytropic equation of state. The timescales considered are long compared to the local disk rotation time.

Results. The scalings relate to dynamics that are radially geostrophic and vertically hydrostatic. A potential vorticity quantity emerges and is shown to be conserved in a Lagrangian sense. Uniform potential vorticity linear solutions are explored and the theory is shown to contain an incarnation of the strato-rotational instability under channel flow conditions. Linearized solutions of a single defect on an infinite domain are developed and shown to support a propagating Rossby edgewave. Linear non-uniform potential vorticity solutions are also developed and shown to be similar in some respects to the dynamics of strictly two-dimensional inviscid flows. The relationship of the scalings and some of the resulting dynamics are considered with respect to other approximations employed in the literature. Based on the framework of this theory, arguments based on geophysical notions are presented to support the assertion that the strato-rotational instability is in a generic class of barotropic/baroclinic potential vorticity instabilities. Extensions of this formalism are also proposed.

Conclusions. The shallow water formulation achieved by the asymptotic theory developed here opens a new approach in studying disk dynamics.
\end{abstract}

Key words. circumstellar matter - instabilities - hydrodynamics - waves

\section{Introduction}

The question of the formation of coherent structures in Keplerian disks has received increased attention in the past few years. Their importance has been recognized in playing a role in a number of processes including angular momentum transport and the accumulation of dust in the process of planet building. These coherent structures are persistent vortices and understanding their generation, structure, dynamics and lifetimes has been the focus of a number of studies both in 2D (e.g. Godon \& Livio 1999; Bracco et al. 1999; Umurhan \& Regev 2004; Johnson \& Gammie 2005; Petersen et al. 2007a, 2007b; Bodo et al. 2007; Lithwick 2007a) and recent 3D studies (Barranco \& Marcus 2005; Lithwick 2007b).

There are many facets to the nature of vortices in strongly sheared and rotating environments like astrophysical disks. One thing that most two-dimensional studies have shown, and which are in qualitative agreement with each other, is that from a patch of noisy initial conditions anti-cyclonic vortices naturally emerge and these structures are usually the sole occupants of radially demarcated zones. This generic phenomenon is observed in 2D shearing box simulations (Umurhan \& Regev 2004; Johnson \& Gammie 2005; Lithwick 2007a) and 2D cylindrical studies as well (Godon \& Livio 1999; Bracco et al. 1999; Petersen et al. 2007a,b; Bodo et al. 2007). In all of these examples a vorticity (or potential vorticity) quantity primarily characterizes the flow dynamics. This vorticity may evolve (nearly) conservatively in a Lagrangian sense (e.g. Godon \& Livio 1999; Bracco et al. 2000; Umurhan \& Regev 2004; Lithwick 2007a) or may have a source term due to baroclinicity leading to, for example, Rossby wave-like dynamics (i.e. wave generation due to radial gradients in temperature or entropy as in Lovelace et al. 1999; Li et al. 2000; Klahr \& Bodenheimer 2003; Petersen et al. 2007a,b), or other wave generation processes of some type (see e.g. the compressible studies of Johnson \& Gammie 2005; Bodo et al. 2007).

The dynamics of vortices in 3D simulations is less clear and the picture of what is occurring under these circumstances is only now emerging. Lithwick (2007b) has demonstrated that columnar anticyclonic vortices can manifest themselves in 3D shearing box simulations with no external gravity. It was also shown in that study that the survival of the vortex column depends on the height of the column itself - if it is too large secondary instabilities may develop causing a toppling over of the structure, otherwise, if it is short enough the structure continues to persist. In an anelastic stratified fluid model evolved in a shearing box with the presence of an external disk-vertical component of gravity, Barranco \& Marcus (2005) show that columnar anticyclonic vortices show a tendency to breakdown but are replaced with anticyclonic structures of restricted vertical extent 
located away from the disk midplane. The way that this process emerges is subtle and a full mechanical understanding of it still awaits - perhaps aided by a shallow water model description.

Shallow water models are effective at capturing salient physical effects involving stratification without having to resort to full $3 \mathrm{D}$ analyses and simulations. In this way they have proven to be indispensible tools in furthering our physical understanding of oceanic and atmospheric flows (Pedlosky 1987) and have recently been used in studies of exoplanet atmospheres (Langton \& Laughlin 2007; Cho et al. 2007).

In a series of studies (Balmforth et al. 1992, 1993; Balmforth et al. 1995; Balmforth \& Spiege 1996) a shallow water formalism was presented and analyzed to study the axisymmetric structure and dynamics of thin cold disks both with and without the effects of self-gravity. This formalism proved to be especially effective at capturing and describing the dynamical response of self-gravitating disk systems. In a limit akin to a shallow water reduction, the linearized equations of motion of small sections of accretion tori have been shown to lead to interesting non-axisymmetric dynamics including processes such as the Papaloizou-Pringle instability (PPI, Papalouizou \& Pringle 1984, 1985; Goldreich et al.1986). We shall return to this in the Discussion section.

Owing to the ubiquity of (potential) vortex dynamics in $2 \mathrm{D}$ and the appearance of long-lived coherent structures in 3D simulations it is worthwhile to develop a framework to better understand the formation of vortices in $3 \mathrm{D}$ scenarios by comparison to the processes leading to $2 \mathrm{D}$ vortex structures. A shallow water theory appropriate for non-axisymmetric disturbances of an accretion disk should serve a critical intermediary role in understanding the physical development of such processes. In this context, meteorological studies ought not to be overlooked: potential vorticity dynamics (sometimes referred to in this work as "PV") and its role in the generation and development of planetary storms in the upper atmosphere have been long appreciated (Hoskins et al. 1985; Bishop \& Davies 1994, and many others).

The road to such a shallow water formalism is guided in part by the results thus far established in the literature. Especially important is the tendency seen in 2D cylindrical studies for (anticyclonic) vortices to either fill out or be the sole occupant of the azimuthal scale of their radially restricted domain (unambiguous examples of this may be found in Godon \& Livio 1999; Petersen et al. 2007a,b). When viewed in a reference frame moving with a steady vortex the scale of the azimuthal velocities (" $\tilde{\mathcal{U}}$ ") are larger than the corresponding radial velocities in proportion to the ratio of the azimuthal to radial length scales of the vortex structure. Assuming that the azimuthal scale is the radial position where the vortex is found (" $R_{0}$ ") then the circulation time of these vortex structures is $\sim R_{0} / \tilde{\mathcal{U}}$. Such vortices in thin (cold) disks, whose azimuthal velocity scales are no greater than the typical sound speeds, will have time scales which are $1 / \epsilon$ longer than the typical rotation time of the disk at the location of the vortex, where $\epsilon$ is the ratio of the local soundspeed to the local Keplerian velocity. In typical cold disks this number is on the order of $1 / 20$.

We therefore reanalyze the full equations of motion (in cylindrical coordinates) by considering the dynamics taking place in an annular section of the disk instead of the usual box section known as the Shearing Box (Goldreich \& Lynden-Bell 1965). The radial and vertical scales are scaled by the disk height, $H_{0}=\epsilon R_{0}$, where (as above) $R_{0}$ is the radial scale of the disk. The azimuthal length scale considered is the full $2 \pi R_{0}$ complement of the disk. The velocity perturbations sitting atop the basic flow (which we assume to be Keplerian, i.e. rotationally supported) are such that the azimuthal velocity fluctuations scale with the local soundspeed while the radial and vertical velocities are much smaller than this by order $\epsilon$. Most importantly, temporal variations do not scale by the usual rotation time scale (given by the inverse of the Keplerian rotation frequency $\bar{\Omega}_{0}$ of the disk at $R_{0}$ ) but on a time scale which is $1 / \epsilon$ longer. In order to affect a shallow water reduction in a transparent way without entirely dispensing with the compressible nature of the gas itself we assume here that the disk gas behaves homentropically where, in particular, we assume a polytropic equation of state characterized by the index $\gamma$ (as in Salby 1989; Cho et al. 2007, and see also the earlier studies of Papalouizou \& Pringle 1984, 1985; Goldreich et al. 1986).

These adopted scalings, especially the slow timescale, lead to conditions in which disturbances are vertically hydrostatic. It also follows that disturbances are geostrophic but only in the radial direction. This means to say that the dynamics are everywhere in radial geostrophic balance, i.e. radial pressure gradients balancing Coriolis effects. These circumstances are similar to the situation encountered resulting from similar scalings of the standard equations of the shearing box (e.g. the Large-Shearing Box limit in Umurhan \& Regev 2004; Umurhan 2006). This type of leading order radial geostrophic balance has been encountered in prior related work as well (Balmforth \& Spiegel 1996; Barranco et al. 2000).

The main result of this analysis then shows that in this asymptotic limit there exists a potential vorticity quantity which is conserved in a Lagrangian sense in the radial and azimuthal directions. In other words we have

$\frac{\mathrm{D} \Xi}{\mathrm{D} t}=0, \quad \Xi=\frac{\Omega_{0}(2-q)+\partial_{x} v}{h^{\frac{\gamma+1}{\gamma-1}}}$,

where $\Xi$ is the potential vorticity. The azimuthal velocity $v$ represents perturbations atop the basic (local) Keplerian flow and where $h(x, y, t)$ is the height of the disk measured from the disk midplane. The Lagrangian time derivative operation is restricted to the two horizontal directions:

$\frac{\mathrm{D}}{\mathrm{D} t} \equiv \partial_{t}+u \partial_{x}+\left(v-q \Omega_{0} x\right) \partial_{y}$

and with $\Omega_{0}$ measuring the local rotation rate of the disk (here it is scaled to 1). The basic Keplerian flow is given by $-q \Omega_{0} x$ in which $x$ is the local (Cartesion) radial coordinate and $q=3 / 2$. The azimuthal and radial velocities are given diagnostically in terms of the height of the disk,

$v=\mathcal{V}(h) \equiv \frac{\Omega_{0}}{4} \partial_{x} h^{2}$,

$u=\mathcal{P}^{-1} \mathcal{F}(h)$,

in which the operators $\mathcal{P}$ and $\mathcal{F}$ are

$$
\begin{aligned}
\mathcal{F}(h) & \equiv\left(\partial_{x} \mathcal{V}-q \Omega_{0}-2 \Omega_{0}\right) \cdot \partial_{y} Q_{h}+2 \partial_{x}\left(\frac{\gamma-1}{\gamma+1} Q_{h} \partial_{y} \mathcal{V}\right), \\
\mathcal{P} & \equiv \Omega_{0}^{2} 2(2-q)-2 \Omega_{0} \mathcal{V} \cdot \partial_{x}-2 \partial_{x}\left(\frac{\gamma-1}{\gamma+1} Q_{h} \partial_{x}\right)
\end{aligned}
$$

The function $Q_{h} \equiv\left(\Omega_{0}^{2} / 2\right) h^{2}$, the radial and horizontal velocities are derived to be related to the vertical linearly varying velocity, (i.e. $w=\Omega_{z} z$, where $\Omega_{z}=\Omega_{z}(x, y, t)$ ) via a quasi-anelastic equation of state,

$\partial_{x} u+\partial_{y} v+\frac{\gamma+1}{\gamma-1} \partial_{z} w=0$ 
As such, these equations describe varicose disturbances with respect to the disk midplane (cf. usage in Balmforth et al. 1992) since the vertical velocity has odd symmetry with respect to the midplane.

We use the set (1-5) to solve a number of example problems. A linearized study of uniform potential vorticity disturbances in a channel flow configuration is presented. Disturbances with only one solid boundary on a semi-infinite domain manifest as a single edgewave (Goldreich et al. 1986) propagating along the wall. Disturbances on a finite domain sandwiched by two solid boundaries manifest as two edgewaves - each associated with their respective boundary. These objects are similar to the edgewaves discussed by Goldreich et al. (1986) rationalizing the Papalouizou-Pringle instability (Papalouizou \& Pringle 1985). These edgewaves become unstable if the width of the channel lies between a narrow band of values less than the height of the fluid layer. For these circumstances we understand this instability as being the incarnation of the stratorotational instability discussed by Dubrulle et al. (2004).

Linearized disturbances of a radially infinite domain with a single global potential vorticity defect appear as a Rossby edgewave propagating along the defect. When the potential vorticity on either side of the defect becomes infinite (negatively) the Rossby edgewave limits to the edgewave solution associated with the semi-infinite domain discussed above. We therefore rationalize the use of a solid boundary as representing this limiting form.

We also investigate the dynamics of non-uniform potential vorticity disturbances subject to the popular shearing-sheet boundary conditions (Goldreich \& Lynden-Bell 1965). We find that the potential vorticity evolves in ways that are very similar to 2D vorticity disturbances as in Chagelishvilli et al. (2003) and Umurhan \& Regev (2004). We find that potential vorticity waves which are leading (in character) show strong transient growth thanks to the Orr-tilting mechanism (Schmid \& Henningson 2000) which is inline with the 2D studies cited as well as being inline with the general tendency for this to happen in 3D linearized disturbances as studied by Korycansky (1992) and Tevzadze et al. (2004).

This work is organized as follows. In Sect. 2 we develop the derivation of (1-5). In Sects. 3-5 we present the linear results of the study of the uniform, single defect and non-uniform potential vorticity disturbances (respectively). In Sect. 6 we, summarize our results, discuss the relationship of the scaling assumptions employed here in context of other approximations employed in the literature, consider some of the dynamical results with respect to other published results and we discuss possible future directions and extensions. We suggest that despite the use of artificial boundary conditions in the classical SRI analysis, that the instability could be possible in real disks supporting regions of oppositely signed potential vorticity gradients.

\section{Scaling analysis and derivation of the reduced equations}

The full equations of motion

$$
\begin{aligned}
\frac{\mathrm{d} \rho}{\mathrm{d} T}+\rho \nabla \cdot \boldsymbol{U} & =0, \\
\frac{\mathrm{d} \boldsymbol{U}}{\mathrm{d} T} & =\frac{1}{\rho} \nabla P-\nabla \Phi, \\
\frac{\mathrm{d} S}{\mathrm{~d} T} & =0,
\end{aligned}
$$

are considered in cylindrical coordinates $(R, \phi, Z)$ where $\boldsymbol{U} \equiv$ $\left(U_{R}, U_{\phi}, U_{Z}\right)$ and where the entropy $S \equiv C_{V} \ln P / \rho^{\gamma}$ with $\gamma=$ $C_{P} / C_{V}$, i.e. the ratio of specific heats. The external potential comes from the central gravitating source and has the form

$$
\Phi \equiv \frac{\bar{\Omega}_{0}^{2} R_{0}^{2}}{\left(\frac{R^{2}}{R_{0}^{2}}+\frac{Z^{2}}{R_{0}^{2}}\right)^{1 / 2}},
$$

in which the frequency $\bar{\Omega}_{0}=\sqrt{G M / R_{0}^{3}}$ is the Keplerian rotation rate at the fiducial radius $R=R_{0}$. The derivation of the equations follows that of Umurhan \& Regev (2004). We shall consider a region centered around the radial point $R=R_{0}$ and $Z=0$. We transform our reference frame so that we are rotating with frequency $\bar{\Omega}_{0}$ appropriate for the radial point $R_{0}$. We begin by stating a number of scalings for the various quantities involved. We assume that at this point the density of the fluid has a scale $\bar{\rho}$ and that the pressure scales as $\bar{\rho} c_{\mathrm{s}}^{2}$ where $c_{\mathrm{s}}$ is the sound speed. Furthermore, we define the non-dimensional parameter

$\epsilon=\frac{c_{\mathrm{s}}}{v_{k}}, \quad v_{k} \equiv \bar{\Omega}_{0} R_{0}$,

where $v_{k}$ is the Keplerian rotation velocity at $R=R_{0}$. We shall assume that $\epsilon$ is less than 1 and "small". We shall consider radial and vertical extent which scale as $\epsilon R_{0}$ - thus this is formally a box-like region in these directions. For the azimuthal scales we shall assume them to be of the order $R_{0}$. In this sense the domain represents a thin annulus instead of a classic small box like the shearing box.

The departures from the classic shearing box equation derivation (e.g. as in Umurhan \& Regev 2004) appears at this point. Following the heuristic observations and arguments proffered in the Introduction, we assume that time scales as $1 / \epsilon \bar{\Omega}_{0}$ and that the vertical and radial velocities (in the frame moving with frequency $\bar{\Omega}_{0}$ ) scale as $\epsilon c_{\mathrm{s}}$ while the azimuthal velocity fluctuations scale as the usual $c_{\mathrm{s}}$. In summary we assume the following scalings

$$
\begin{aligned}
R & \rightarrow R_{0}+\epsilon R_{0} x, Z \rightarrow \epsilon R_{0} z, \\
\phi & \rightarrow y-\bar{\Omega}_{0} t, \quad T \rightarrow t /\left(\epsilon \bar{\Omega}_{0}\right), \\
U_{R} & \rightarrow \epsilon c_{\mathrm{s}} u, \quad U_{z} \rightarrow \epsilon c_{\mathrm{s}} w, \quad U_{\phi} \rightarrow v_{k}+c_{\mathrm{s}} v_{y}, \\
\rho & \rightarrow \bar{\rho} \rho, \quad P \rightarrow \bar{\rho} c_{\mathrm{s}}^{2} p .
\end{aligned}
$$

The variables $p, \rho, t, x, z, y, u, w, v_{y}$ appearing on the righthandsides of all transformations are understood to be order 1 nondimensional quantities corresponding to their dimensional counterparts. Inserting these expansions into the governing equations of motion expressed in the rotating frame yields the following set of equations at lowest order in $\epsilon$,

$$
\begin{aligned}
\frac{\mathrm{d} \rho}{\mathrm{d} t}+\rho \nabla \cdot \boldsymbol{u} & =0+O(\epsilon) \\
-2 \Omega_{0} v_{y} & =-\frac{1}{\rho} \partial_{x} p+2 \Omega_{0}^{2} q x+O\left(\epsilon^{2}\right) \\
\frac{\mathrm{d} v_{y}}{\mathrm{~d} t}+2 \Omega_{0} u & =-\frac{1}{\rho} \partial_{y} p+O\left(\epsilon^{2}\right) \\
0 & =-\frac{1}{\rho} \partial_{z} p-\Omega_{0}^{2} z+O\left(\epsilon^{2}\right) \\
\frac{\mathrm{d} s}{\mathrm{~d} t} & =0+O(\epsilon)
\end{aligned}
$$


where $s \equiv \ln p / \rho^{\gamma}, q=3 / 2$ and $\Omega_{0}=1$. The (Cartesian) time derivative operation is given to be

$\frac{\mathrm{d}}{\mathrm{d} t}=\partial_{t}+u \partial_{x}+v_{y} \partial_{y}+w \partial_{z}$

while the divergence operation is

$\nabla \cdot \boldsymbol{u}=\partial_{x} u+\partial_{y} v_{y}+\partial_{z} w$.

We shall assume that the fluid behaves as a barotrope where, in particular, we assume that it behaves like a polytropic gas, i.e.

$p=K \rho^{\gamma}$,

and consequently it means that the fluid layer is homentropic. This is to say that the fluid layer is characterized by a constant entropy given to be $s=\ln K$. We may now exactly solve for (13) by defining the fluid enthalpy $Q$ (as in Goldreich et al. 1986),

$\mathrm{d} Q=\frac{1}{\rho} \mathrm{d} P=K \frac{\gamma}{\gamma-1} \mathrm{~d} \rho^{\gamma-1}$.

In terms of the enthalpy (13) is simply

$\partial_{z} Q=-\Omega_{0}^{2} z$

yielding the solution

$Q=\frac{1}{2} \Omega_{0}^{2}\left(h^{2}-z^{2}\right)$.

The height $h=h(x, y, t)$ defines where the fluid density and pressure go to zero and is a function of the horizontal coordinates and time.

The entropy Eq. (14), the equation of continuity (10), the polytropic gas assumption and the solution to $Q(18)$, all taken together yield

$\frac{\mathrm{d} Q}{\mathrm{~d} t}+(\gamma-1) Q \nabla \cdot \boldsymbol{u}=0$

For the solutions we shall examine here, as done in the derivation of shallow water equations, we state the ansatz that the horizontal velocities have no $z$-dependencies while the vertical velocity has a linear $z$-dependence, i.e.

$u=u(x, y, t), \quad v_{y}=v_{y}(x, y, t), \quad w=\Omega_{z}(x, y, t) z$.

By choosing this form for the vertical velocity we are restricting attention to varicose disturbances of this annular disk section. Explicitly inserting the solution form (18) into (19) and making use of the ansatz for $w$ we find

$$
\begin{aligned}
& \frac{\mathrm{D}}{\mathrm{D} t}\left(\frac{1}{2} \Omega_{0}^{2} h^{2}\right)-\Omega_{z} \Omega_{0}^{2} z^{2} \\
& \quad(\gamma-1) \frac{1}{2} \Omega_{0}^{2}\left(h^{2}-z^{2}\right)\left(\partial_{x} u+\partial_{y} v_{y}+\Omega_{z}\right)=0,
\end{aligned}
$$

in which

$$
\frac{\mathrm{D}}{\mathrm{D} t} \equiv \partial_{t}+u \partial_{x}+v_{y} \partial_{y}
$$

On account of their linear independence, we equate to zero the coefficients of each power of $z$ appearing in (21). In this particular situation this yields two equations, the first is the equation for the height $h$

$$
\frac{\mathrm{D} h}{\mathrm{D} t}=h \Omega_{z},
$$

while the second is an expression resembling an anelastic condition

$\partial_{x} u+\partial_{y} v_{y}+\frac{\gamma+1}{\gamma-1} \Omega_{z}=0$.

We note that the limit $\gamma \rightarrow \infty$ recovers the condition of incompressible fluid flow. Addressing (11) we rewrite the azimuthal velocity $v_{y}=-q \Omega_{0} x+v$ to find this equation appearing now as the geostrophic balance discussed in the Introduction,

$-2 \Omega_{0} v=-\partial_{x} Q$,

while (12) now appears

$\frac{\mathrm{D} v}{\mathrm{D} t}+\Omega_{0}(2-q) u=-\partial_{y} Q$.

Note that the time derivative looks like

$\frac{\mathrm{D}}{\mathrm{D} t}=\left(\partial_{t}-q \Omega_{0} x \partial_{y}\right)+u \partial_{x}+v \partial_{y}$.

The set of Eqs. (22-25) form the basic equations of this asymptotic limit.

We may proceed even further and develop a potential vorticity equation. We begin by operating on (24) with $\partial_{y}$ and subtracting from this the result of operating on (25) with $\partial_{x}$ leaving

$\frac{\mathrm{D} \partial_{x} v}{\mathrm{D} t}+\left[\Omega_{0}(2-q)+\partial_{x} v\right]\left(\partial_{x} u+\partial_{y} v\right)=0$.

Using (23) in the above and combining the result with (22) finally results in (1).

The quantity $\Xi$ is the potential vorticity and (1) is the analogous Taylor-Proudman theorem for this shallow water system (cf. Pedlosky 1987; Cho et al. 2007). We may further isolate the radial velocity in terms of $h$. We do this by (i) multiplying (22) by $h \Omega_{0}^{2} / 2$; (ii) operating on the resulting equation with the partial derivative operation $\partial_{x}$; (iii) subtracting the resulting equation from (25); (iv) reordering the result and using (24) results in the diagnostic equations for $u$ and $v$ found in (2-4).

\section{Constant uniform potential vorticity solutions}

We consider the dynamics emerging from a situation where $\Xi$ is uniform and constant. We imagine that we disturb a uniform equilibrium $h=h_{0}$. Thus this means that the uniform potential vorticity state is given by

$\Xi_{0}=\Omega_{0} \frac{2-q}{h_{0}^{\frac{\gamma+1}{\gamma-1}}}$.

Through the use of (24) we find that the equation to be solved is

$\Omega_{0}(2-q)+\Omega_{0} \partial_{x}^{2}\left(\frac{1}{4} h^{2}\right)-\Xi_{0} h^{\frac{\gamma+1}{\gamma-1}}=0$,

subject to specified boundary conditions. Given that we will be considering disturbances around the uniform state we define a new quantity $\Theta$ as

$\theta=\frac{h-h_{0}}{h_{0}}$

Thus, the potential vorticity Eq. (28) is written more simply as

$\frac{1}{2} h_{0}^{2} \partial_{x}^{2}(1+\theta)^{2}+2(2-q)\left[1-(1+\theta)^{\frac{\gamma+1}{\gamma-1}}\right]=0$. 
We shall consider here only two possible boundary conditions. The first is that the normal velocity is zero at the position $x=0$ and that the pressure remain fixed as $x \rightarrow \infty$ which translates to requiring $h \rightarrow h_{0}$. We shall refer to this scenario as the "semiinfinite domain". Referring to (25) the condition on the normal velocity is the same as requiring

$\left(\partial_{t}-q \Omega_{0} x \partial_{y}\right) v+v \partial_{y} v+\frac{1}{2} \partial_{y} \Omega_{0}^{2}(1+\theta)^{2}=0$,

at $x=0$ and where $v=\Omega_{0} \frac{1}{4} \partial_{x}(1+\theta)^{2}$. The second set of boundary condition is that we require the normal velocities be zero at the positions $x= \pm \Delta$. In other words, we require that (30) be satisfied at those boundaries. We refer to this as the "channel flow".

We consider linear disturbances for $\theta$. The governing equation for the disturbance PV, $\Xi^{\prime}$, becomes

$\Xi^{\prime} \equiv h_{0}^{2} \partial_{x}^{2} \theta-2(2-q) \frac{\gamma+1}{\gamma-1} \theta=0$.

The normal velocity condition at the boundaries becomes

$\left(\partial_{t}-q \Omega_{0} x \partial_{y}\right) \partial_{x} \theta+2 \Omega_{0} \partial_{y} \theta=-2(2-q) u=0$.

Semi-infinite domain. The solution appropriate to the semiinfinite domain is

$\Theta=-\frac{A(y, t)}{\kappa} \mathrm{e}^{-\kappa x}, \quad \kappa^{2}=\frac{2(2-q)}{h_{0}^{2}} \frac{\gamma+1}{\gamma-1}$,

where we select the $\kappa>0$ solution. The amplitude time evolution of the amplitude $A$ is obtained from the velocity boundary condition (32),

$\partial_{t} A+2 \Omega_{0} \kappa^{-1} \partial_{y} A=0$.

Making the normal mode ansatz of the form $A(y, t) \sim$ $\exp i \alpha(y-c t)$ where $\alpha$ is the streamwise wavenumber, we find that the wavespeed is given by

$c=-2 \Omega_{0} / \kappa$.

Since $c<0$, it follows that these are wave patterns which propagate in the negative $y$ direction, that is to say, against the mean flow.

Channel flow. Under channel flow conditions the normalmode solution of $\theta$ is

$\theta=[A \cosh \kappa(x+\Delta)-B \cosh \kappa(x-\Delta)] \mathrm{e}^{i \alpha(y-c t)}$.

Enforcing the linear boundary conditions (32) at the points $x=$ $\pm \Delta$ using the solution (36) we find

$-\left(c+q \Omega_{0} \Delta\right) A \kappa \sinh 2 \kappa \Delta+2 \Omega_{0}(A \cosh 2 \kappa \Delta-B)=0$,

at $x=-\Delta$ and

$-\left(c-q \Omega_{0} \Delta\right) B \kappa \sinh 2 \kappa \Delta+2 \Omega_{0}(A-B \cosh 2 \kappa \Delta)=0$,

at $x=\Delta$. These two simultaneous equations are satisfied nontrivially if the wave speed $c$ satisfies,

$c^{2}=q^{2} \Omega_{0}^{2} \Delta^{2}+\frac{4 \Omega_{0}^{2}}{\kappa^{2}}(1-q \kappa \Delta \operatorname{coth} 2 \kappa \Delta)$.

The wavespeeds approach the local mean flow speed of the respective boundaries as the separation between the walls becomes large, i.e.

$\lim _{\Delta \rightarrow \infty} c^{2}=\Omega_{0}^{2} \Delta^{2} q^{2}$.

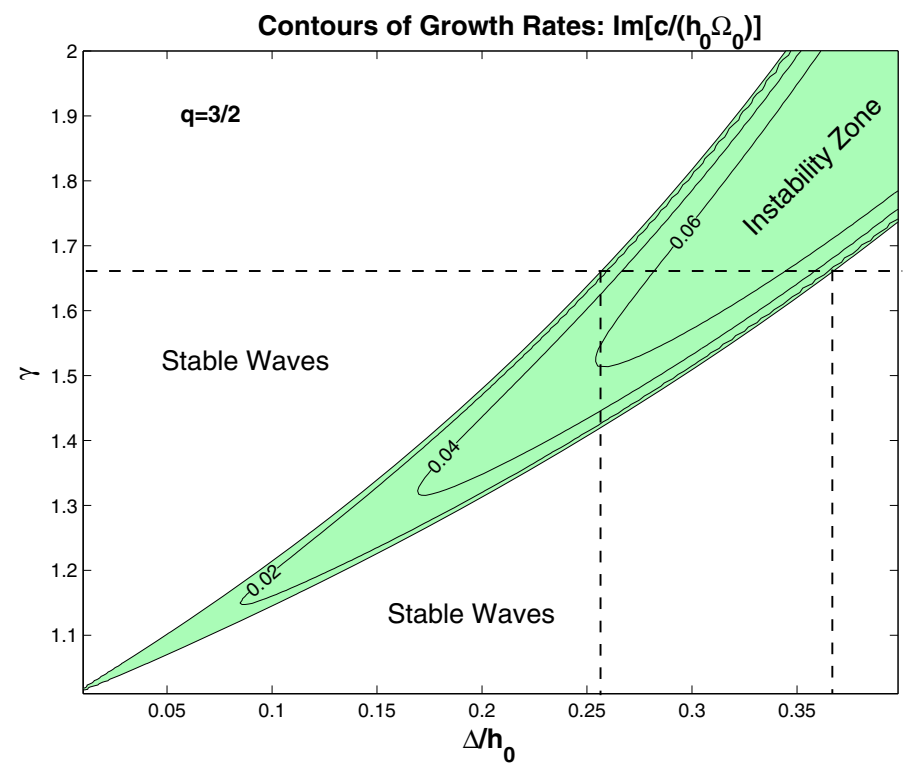

Fig. 1. Contours of growth rates of the SRI as a function of $\gamma$ and $\Delta / h_{0}$. The growth rates are scaled by $\Omega_{0} h_{0}$. The behavior for $q=3 / 2$ is shown. The shaded region corresponds to the instability band. Horizontal line designating $\gamma=5 / 3$ is also shown.

On the other hand, when the wall separation becomes small the wavespeeds limit to

$\lim _{\Delta \rightarrow 0} c^{2}=h_{0}^{2} \Omega_{0}^{2} \frac{\gamma-1}{\gamma+1}$

Examining the wavespeed prescription (39) in the case where there is no shear, i.e. $q=0$, shows that there is no possibility of instability. However, we do see that there seems to exist a band of instability in the gap width $\Delta$ for values of $q>0$. In Fig. 1 we plot the region of instability and growth rate as a function of a variety of parameters. We see that range in $\Delta$ for which instability is predicted is less than $h_{0}$ : for instance, for $\gamma=5 / 3$ instability is expected approximately only when $0.26 h_{0}<\Delta<$ $0.37 h_{0}$. The range widens as $\gamma$ increases but is still less than $h_{0}$ for physically reasonable values of $\gamma$. Note that the thickness of the channel is measured by $2 \Delta$.

\section{Normal-modes of an infinite domain with a single PV defect}

In this section we consider the response of an infinite domain separated by a single defect in the potential vorticity. For simplicity we again treat $h_{0}$ as constant. Therefore, the defect appears in this case as a jump in the basic state shear, i.e.

$q=\left\{\begin{array}{ll}q_{+}, & x>0, \\ q_{-}, & x<0 .\end{array}, \quad V_{0}= \begin{cases}-\Omega_{0} q_{+} x, & x>0 \\ -\Omega_{0} q_{-} x, & x<0 .\end{cases}\right.$

Where $V_{0}$ is the steady state uniform shear. This formulation ensures that the background velocity is everywhere continuous. Normal mode disturbances can be developed for this configuration much in the manner developed in the previous section. This means separately solving (31) on either side of the defect 
and then matching the solutions across the interface ${ }^{1}$. Thus, for $x>0$, disturbances which decay as $x \rightarrow \infty$ are

$\theta=A_{+} \mathrm{e}^{i \alpha(y-c t)} \mathrm{e}^{-\kappa_{+} x}, \quad \kappa_{+}^{2}=\frac{2\left(2-q_{+}\right)}{h_{0}^{2}} \cdot \frac{\gamma+1}{\gamma-1}$,

while for $x<0$, disturbances decaying as $x \rightarrow-\infty$ are

$\theta=A_{-} \mathrm{e}^{i \alpha(y-c t)} \mathrm{e}^{\kappa_{-} x}, \quad \kappa_{-}^{2}=\frac{2\left(2-q_{-}\right)}{h_{0}^{2}} \cdot \frac{\gamma+1}{\gamma-1}$,

while in both cases the positive root for $\kappa_{ \pm}$is chosen. Since $\theta$ represents the perturbed height of the disk we require that it be continuous across the interface - this results in $A_{-}=A_{+}=A$. Because we are investigating normal mode disturbances, it also makes physical sense to match the normal velocities across the interface. There are a number of ways to do this but the mathematically simplest is to consider the linearized version of (12),

$\left(\partial_{t}-q \Omega_{0} x \partial_{y}\right) v+\Omega_{0}(2-q) u^{\prime}=-h_{0} \partial_{y} \theta$.

Since the linearized expression of the geostrophic balance relationship is $v^{\prime}=\frac{1}{2} \partial_{x} \theta$, in order for the radial velocity $u^{\prime}$ to be continuous across the defect we must have that

$\left[u^{\prime}\right]_{x \rightarrow 0^{-}}^{x \rightarrow 0^{+}}=\left[\frac{\mathrm{i} \alpha\left(-c-q \Omega_{0} x\right) \partial_{x} \theta+2 \Omega_{0} \mathrm{i} \alpha \theta}{2-q}\right]_{x \rightarrow 0^{-}}^{x \rightarrow 0^{+}}=0$.

Given the solution for $\theta$ on either side of $x=0$, in order for this to be satisfied is if the wavespeed $c$ satisfies the relationship

$c=-2 \Omega_{0} \frac{q_{+}-q_{-}}{\kappa_{+}\left(2-q_{-}\right)+\kappa_{-}\left(2-q_{+}\right)}$.

The wavespeed represents the propagation of a Rossby edgewave in the sense presented in Hoskins et al. (1985). So long as $q_{ \pm}<2$ the edgewave propagates in a negative sense with respect to the coordinate $y$ if $q_{+}>q_{-}$. Stated in another way, when this condition is met the Rossby wave travels against the mean flow.

We note a significant mathematical limit of the wavespeed prescription (47): when $q_{-} \rightarrow-\infty$, hile keeping $q_{+}$finite, we find that

$c \rightarrow-2 \Omega_{0} / \kappa_{+}$.

By identifying $q_{+}$with $q$ it follows from the definition of $\kappa$ (33) that $\kappa_{+}=\kappa$. Hence, we have recovered the wavespeed form for the semi-infinite domain, i.e. (35) in which the edgewave propagates against the local mean flow. This view allows us to interpret the presence of an inner wall boundary condition to be like a PV-defect whose PV on the one side of the defect (e.g. $x<0$ ) greatly exceeds in magnitude the PV of the other side of the defect.

This implication can also be understood by studying (46): when, say, $-q_{-} \gg 1$ then it means that the normal velocity approaching the defect from $0^{+}$should be correspondingly small. In the infinite limit it is equivalent to requiring $u^{\prime}\left(x \rightarrow 0^{+}\right)=0$.

\footnotetext{
1 Another way to look at this would be to argue that if the perturbed height of the disk were to change discontinuously then it would correspond to a delta-function horizontal pressure gradient.
}

\section{Linear, non-uniform potential vorticity solutions}

We consider here linearized solutions to the potential vorticity $\Xi^{\prime}$ when it is not in general zero. This means solving

$\left(\partial_{t}-q \Omega_{0} \partial_{y}\right) \Xi^{\prime}=0$

given the definition of $\Xi^{\prime}$ to be found in (31), this may be rewritten instead as

$\left(\partial_{t}-q \Omega_{0} x \partial_{y}\right)\left[2(2-q) \frac{\gamma+1}{\gamma-1}-h_{0}^{2} \partial_{x}^{2}\right] \theta=0$.

The radial velocity is the solution to

$$
\begin{aligned}
& {\left[2(2-q)-\frac{\gamma-1}{\gamma+1} h_{0}^{2} \partial_{x}^{2}\right] u=} \\
& h_{0}^{2} \Omega_{0}\left[-2(q+2)+\frac{\gamma-1}{\gamma+1} h_{0}^{2} \partial_{x}^{2}\right] \frac{1}{2} \partial_{y} \theta .
\end{aligned}
$$

We shall consider here wave solutions on the classical sheared periodic domain as introduced by Goldreich \& Lynden-Bell (1965). To this end we define a new coordinate system in which the new azimuthal coordinate moves with the local shear

$X=x, \quad Y=y+q \Omega_{0} x t, \quad T=t$,

so that all derivative operations now translate to

$\partial_{t} \rightarrow \partial_{T}, \quad \partial_{x} \rightarrow \partial_{X}+q \Omega_{0} T \partial_{y}, \quad \partial_{y} \rightarrow \partial_{Y}$

Then in the usual way we find the evolution equation for $\Xi^{\prime}$ can now be written as

$\partial_{T}\left[h_{0}^{2}\left(\partial_{X}+q \Omega_{0} T \partial_{Y}\right)^{2}+2(2-q) \frac{\gamma+1}{\gamma-1}\right] \theta=0$.

In this frame we assume Fourier solutions of the form

$\theta=\theta_{k \ell}(T) \mathrm{e}^{i k X+i \ell Y}$,

where $k$ and $\ell$ are wavevectors. The solution to the wave amplitude $\theta_{k \ell}(T)$ is given simply by

$\theta_{k \ell}=\hat{\theta}_{k \ell} \frac{h_{0}^{2} k^{2}+2(2-q) \frac{\gamma+1}{\gamma-1}}{h_{0}^{2}\left(k+q \Omega_{0} \ell T\right)^{2}+2(2-q) \frac{\gamma+1}{\gamma-1}}$,

where $\hat{\theta}_{k \ell}$ is the wave amplitude at time $T=0$. The solution to the aziumuthal velocity may also be expressed as

$v=v_{k \ell} \mathrm{e}^{i k X+\mathrm{i} \ell Y}$,

$v_{k \ell}=-\mathrm{i} \frac{1}{4} \Omega_{0} h_{0}\left(k+q \Omega_{0} \ell T\right) \theta_{k \ell}$.

Similary, representing the solution for $u$ as

$u_{k \ell}(T) \mathrm{e}^{i k X+i \ell Y}$

we have from (51)

$u_{k \ell}=-\mathrm{i} \ell \frac{1}{2} h_{0}^{2} \Omega_{0} \frac{2(2+q)+\frac{\gamma-1}{\gamma+1} h_{0}^{2}\left(k+q \Omega_{0} \ell T\right)^{2}}{2(2-q)+\frac{\gamma-1}{\gamma+1} h_{0}^{2}\left(k+q \Omega_{0} \ell T\right)^{2}} \theta_{k \ell}$.

Long time evolution of the solution to $\theta_{k \ell}$ and $u_{k \ell}$ shows decay as $1 / T^{2}$ while $v_{k \ell}$ decays as $1 / T$. If one restricts attention to solutions where $T>0$, leading waves, i.e. those in which $k \ell<0$, will show a maximum in the amplitude of $\theta_{k \ell}$ when $T_{\max }=k /\left(q \Omega_{0} \ell\right)$. 
The ratio of the amplitudes of these trailing waves to their initial amplitude is

$\frac{\theta_{k \ell}\left(T_{\max }\right)}{\theta_{k \ell}(0)}=1+\frac{h_{0}^{2} k^{2}}{2(2-q)} \cdot \frac{\gamma-1}{\gamma+1}$,

showing that amplification is greater as $k$ increases. Furthermore, at $T_{\max }$ we see that $v_{k \ell}=0$.

On the other hand, trailing waves, i.e. those in which $k \ell>0$, show monotonic decay for all $T>0$. Examples of this sort of behavior is well-known for the evolution of two dimensional vorticity perturbations (e.g. Chagelishvilli et al. 2003; Umurhan \& Regev 2004) and three-dimensional disturbances (Knobloch 1984; Korycansky 1992; Sternberg 2005; Balbus \& Hawley 2006).

\section{Discussion and summary}

In 2D inviscid, incompressible vortex flow in a background linear shear profile, the vorticity is a quantity which is conserved in a Lagrangian sense. In terms of the non-dimensional quantities we have come to use in this study, this means to say that that

$\left[\partial_{t}+u \partial_{x}+\left(v-q \Omega_{0} x\right) \partial_{y}\right] \omega=0$,

in which the vorticity $\omega$ may be expressed in terms of a streamfunction $\psi$ according to $\omega=\left(\partial_{x}^{2}+\partial_{y}^{2}\right) \psi$. The radial and azimuthal velocities are then given by $u=\partial_{y} \psi, v_{y}=-\partial_{x} \psi$. By construction the $u$ and $v_{y}$ velocities satisfy the criterion of incompressibility.

By comparison, the asymptotic system we have developed here, representing the varicose disturbances of an annular section of a cold rotationally balanced accretion disk containing a homentropic gas, has a similar form. In other words it means to say that the corresponding Lagrangian conserved quantity is the potential vorticity $\Xi$, i.e.

$\left[\partial_{t}+u \partial_{x}+\left(v-q \Omega_{0} x\right) \partial_{y}\right] \Xi=0$.

The potential vorticity is related to the disk height $h$ according to

$\Xi=\frac{\Omega_{0}(2-q)+\partial_{x} \mathcal{V}(h)}{h^{\frac{\gamma+1}{\gamma-1}}}$,

where $\gamma$ is the polytropic index characterizing the homentropic layer under consideration. The radial and vertical velocities are given by

$v=\mathcal{V}(h), \quad u=\mathcal{P}^{-1} \mathcal{F}(h)$,

in which the operators appearing above are given in (2-4). The disk height $h$ is, in this case, analogous to the streamfunction in classical 2D inviscid incompressible theory. Unlike the latter, the horizontal velocities reflect the dynamics of a quasi-anelastic relationship of the flow quantities in $3 \mathrm{D}$, i.e.

$\partial_{x} u+\partial_{y} v_{y}+\frac{\gamma+1}{\gamma-1} \Omega_{z}=0$,

where in general the vertical velocity is given as $w=\Omega_{z} z$. The relationships (61-63) including the definition of the operators (2-4) are self-contained.

Note that the quantity $\Omega_{z}$ does not play a direct role in the evolution of the potential vorticity. Its meaning can be understood by noting that the expressions establishing the relationships between the horizontal velocities and the disk height $h$ are
Table 1. Comparison of the various scalings employed that result in some of the approximations used in the literature to study small sections of accretion disks.

\begin{tabular}{lccccc}
\hline \hline Quantity & SSB & LSB & $\begin{array}{c}\text { Elongated } \\
\text { vortices }\end{array}$ & $\begin{array}{c}\text { Anelastic } \\
\text { model }\end{array}$ & $\begin{array}{c}\text { This } \\
\text { work }\end{array}$ \\
\hline $\begin{array}{l}\epsilon \equiv c_{\mathrm{s}} / v_{k} \\
\delta \text { : Mach \# }\end{array}$ & $\ll \epsilon$ & $\begin{array}{c}\ll 1 \\
\sim 1\end{array}$ & $\begin{array}{c}\ll \\
\text { : }\end{array}$ & $\begin{array}{c}\ll 1 \\
\epsilon \ll \delta \ll 1\end{array}$ & $\sim 1$ \\
\hline $\begin{array}{l}\text { azimuthal } \\
\text { scales }\end{array}$ & $\delta R_{0}$ & $\epsilon R_{0}$ & $\epsilon R_{0}$ & $\delta \epsilon R_{0}$ & $R_{0}$ \\
$\begin{array}{l}\text { vertical } \\
\text { scales }\end{array}$ & $\delta R_{0}$ & $\epsilon R_{0}$ & $\epsilon R_{0}$ & $\delta \epsilon R_{0}$ & $\epsilon R_{0}$ \\
$\begin{array}{l}\text { radial } \\
\text { scales }\end{array}$ & $\delta R_{0}$ & $\epsilon R_{0}$ & $\delta \epsilon R_{0}$ & $\delta \epsilon R_{0}$ & $\epsilon R_{0}$ \\
$\begin{array}{l}\text { azimuthal } \\
\text { speeds }\end{array}$ & $\delta c_{s}$ & $c_{s}$ & $\delta c_{s}$ & $\delta c_{s}$ & $c_{s}$ \\
$\begin{array}{l}\text { vertical } \\
\text { speeds }\end{array}$ & $\delta c_{s}$ & $c_{s}$ & $\delta c_{s}$ & $\delta c_{s}$ & $\epsilon c_{s}$ \\
$\begin{array}{l}\text { radial } \\
\text { speeds }\end{array}$ & $\delta c_{s}$ & $c_{s}$ & $\delta^{2} c_{s}$ & $\delta c_{s}$ & $\epsilon c_{s}$ \\
$\begin{array}{l}\text { temporal } \\
\text { scales }\end{array}$ & $1 / \bar{\Omega}_{0}$ & $1 / \bar{\Omega}_{0}$ & $1 /\left(\delta \bar{\Omega}_{0}\right)$ & $1 / \bar{\Omega}_{0}$ & $1 /\left(\epsilon \bar{\Omega}_{0}\right)$ \\
$\begin{array}{l}\text { dynamical } \\
\text { pressure }\end{array}$ & $\delta \bar{\rho} c_{s}^{2}$ & $\bar{\rho} c_{s}^{2}$ & $\delta^{2} \bar{\rho} c_{s}^{2}$ & $\delta^{2} \bar{\rho} c_{s}^{2}$ & $\bar{\rho} c_{s}^{2}$ \\
$\begin{array}{l}\text { dynamical } \\
\text { density }\end{array}$ & $\bar{\rho}$ & $\bar{\rho}$ & $\delta^{2} \bar{\rho}$ & $\delta^{2} \bar{\rho}$ & $\bar{\rho}$ \\
\hline
\end{tabular}

consistent with the material derivative of the disk height, that is, its role may be understood in terms of the relationship,

$\left[\partial_{t}+u \partial_{x}+\left(v-q \Omega_{0} x\right) \partial_{y}\right] h=w(z=h)=h \Omega_{z}$.

\subsection{Relationship to other approximations}

In Table 1 we present a comparison of the various approximations used to model small sections of accretion disks (i.e. conditions in which $\epsilon \ll 1)$. The four approximations presented are ones used in the literature and we describe them briefly below. In all of the following standard approximations the vertical extent of the disk $H$ is assumed to be smaller than the radial scales of the disk by order $\epsilon-$ that is, $H \sim O\left(\epsilon R_{0}\right)$. Additionally, and within the moving frame assumed throughout these considerations, a Mach number is defined by, $\delta \equiv \mathcal{U} / c_{s}$, where $\mathcal{U}$ is the largest velocity scale encountered in the frame.

- SSB. The small shearing box equations are the classical ones used to investigate the dynamics on very small scale sections of disks. Originally proposed by Goldreich \& Lynden-Bell (1965) they have been the basis of numerous studies pertaining to the question of hydrodynamic disk turbulence (Lesur \& Longaretti 2005) and nature and maintanence of 3D disk vortices (Lithwick 2007b). A scaling analysis (Umurhan \& Regev 2004) shows that these disturbances are assumed to have Mach numbers smaller than the aspect ratio of disks (i.e. $\delta \ll \epsilon$ ). The corresponding length scales of disturbances are smaller than the vertical extent of the disk as well but the time scales of all disturbances are equal to the local disk rotation times (i.e. $\sim 1 / \bar{\Omega}_{0}$ ).

- $L S B$. The so-called large shearing box equations are derived from a scaling analysis similar to those leading to the SSB (Umurhan \& Regev 2004) except that the velocities are 
scaled to have Mach numbers which are order 1 . The corresponding length scales of disturbances compare to the vertical scale of the disk. As with the SSB, the time scales of disturbances equal the local disk rotation times. The resulting equations are the compressible version of the equations of the SSB which also allow for the effects of gravity waves as there is now also an entropy equation which is evolved as well.

- Elongated Vortex. Assuming a flow Mach number which is small, an analysis of a Keplerian disk in which one considers disturbances whose azimuthal and vertical scales compare to the local disk height while the radial length scales are smaller by an order $\delta$ lead to the equations describing elongated vortices (Barranco et al. 2000). Under these circumstances, while the subsonic horizontal and vertical velocities of disturbances scale by the Mach number of the soundspeed (i.e. $\delta c_{\mathrm{s}}$ ) the scale of the radial velocity disturbances are smaller than these by another order of the Mach number. Consequently the time scale of all disturbances are an order $1 / \delta$ longer than the local disk rotation time.

- Anelastic Model. Barranco \& Marcus (2005) present a scaling argument leading to a set equations which we refer to here as the Anelastic Model. Flow quantities are formally assumed to have small Mach numbers (i.e. $\delta \ll 1$ ). Azimuthal and radial disturbance scales are assumed to be an order $\delta$ smaller than the vertical scale of the disk $H$ while the vertical disturbances scales are of the order $H$. The subsonic azimuthal and radial velocity disturbances scale by the Mach number factor of the soundspeed while the vertical velocities are a factor of $\delta$ even smaller than these. Consequently, the time scales of all these vortical disturbances is of the order of the disk rotation time. By assuming that the dynamical pressure and densities scale as they do one arrives at a circumstance in which sound waves are filtered from the equation set resulting in equations of motion describing an anelastic fluid.

The scalings describing the dynamical response of the fluid pressure and density disturbances are also presented in the table for the purpose of comparison. The point to take away from this is that the pressure and density fluctuations are small by order $\delta^{2}$ their respective steady state values in the equations describing elongated vortices and the Anelastic Model as compared to the corresponding pressure and density disturbances considered in this work and for the LSB. The dynamic $\delta$ in the SSB. The small scalings of the thermodynamic quantities affect the filtering out of soundwaves.

The scaling analysis we have employed here to arrive at this shallow water limit have some features similar to the arguments leading to the the Elongated Vortex equations of Barranco et al. (2000). Physically it means that there exists radial geostrophic balance of dynamical quantities - a condition which has been visited and explored in prior studies (Balmforth et al. 1992; Balmforth \& Spiegel 1996). The important feature that the approximations made in this study shares with the arguments leading to the Elongated Vortex equations is that the dynamical timescales of the disturbances are long compared to the local disk rotation time. This allows for an analysis which can focus on the long term properties of vortices under these circumstances free of the effect of soundwaves. Soundwaves and other short time fluctuations in this study have been filtered out by the fact that we are considering time scales which are longer than what typifies these wave phenomena. It is this long timescale view together with the assumption made here that the radial and vertical velocity fluctuations are smaller than the azimuthal ones that ultimately lead to the shallow water theory limit arrived at here.

\subsection{Walls, defects, edgewaves and instability}

In an examination of single PV defect disturbances of an infinite domain we saw that by letting the PV on one side of the defect tend to infinity is mathematically equivalent to replacing the defect with an impenetrable wall. Indeed, the limiting form of the Rossby edgewave wavespeed (47) in the limit where the PV on one side becomes infinite recovers the expression for the wavespeed on the semi-infinite domain (35). It would seem then, at least within the context of this theory, that this equivalency may be a useful conceptual tool. For instance, the use of impenetrable boundary conditions is widely considered to be artificial in most cases other than for terrestrial laboratory flows in a channel. This consensus is especially true in the study of astrophysical disks. Of course as disks no not have delineated solid boundaries, this view is physically justified. However, it is conceivable that disks may have regions in which the radially varying potential vorticity undergoes rapid changes. One obvious candidate region would be the star-disk boundary. If the star rotates significantly sub-Keplerian then the PV in the boundary layer region is likely to be significantly different than the PV immediately outside the boundary layer. The transition between the two can then be viewed as a defect in the disk's PV and in the limit where the PV in the boundary layer greatly exceeds the PV outside, the arguments laid out here could be used to justify replacing the defect with a solid boundary for the purposes of analyzing the response in the extended disk.

With this in mind, we can view the edgewave propagating along the solid boundary as a limiting form of a Rossby edgewave propagating along a defect. It follows that much of the machinery involved with the principle of counterpropagating Rossby waves (CRW), originally proposed in Hoskins et al. (1985) in the context of understanding barotropic/baroclinic instabilities in planetary atmospheres, should carry over to understanding disks as well. In Sect. 3 we presented a normal mode analysis on a finite domain bounded by two impenetrable walls. The instability associated with it is a manifestation of the SRI. In view of the analysis, we see that the two modes appearing under those circumstances are Rossby edgewaves propagating along the boundary walls. Under the right conditions (i.e. wall separation) these edgewaves interact with each other in such a way as to promote an instability in the way outlined and demonstrated in Molemaker et al. (2001) - much in the same way that edgewaves are responsible for the emergence of the PPI (Goldreich et al. 1985, and see below).

Now, given the foregoing argument, the edgewaves associated with the boundaries can be viewed as a limiting form of Rossby edgewaves propagating along a PV defect in which the PV on one side greatly exceeds the other. In this way, the left boundary looks like it has a negative PV gradient while the gradient on the right boundary looks positive which satisfies the two-signed PV-gradient instability condition of Charney \& Stern (1962) which means that the mechanism of the instability can be physically rationalized as arising from the interaction of CRWs (Hoskins et al. 1985; Heifetz et al. 2004). Such instabilities ultimately draw their energy from the background shear - without which the instability vanishes as is the case here. An examination of the SRI in which the channel boundaries are replaced with either PV defects or strongly varying smooth PV profiles is the subject of a future study. 
A real disk probably is rotationally supported all the way through, which would mean that the only way that one could realistically argue for significant changes in its PV profile would be if the steady-state height of the disk changes as a function of the radial coordinate. Such localized annuli will probably introduce some departures from Keplerian flow as well. The possibility of this scenario has been proposed before by Lovelace et al. (1999) and Li et al. (2000) in the context of twodimensional disks characterized by strong radial entropy gradients where they show that Rossby waves turn unstable under suitable circumstances. It is in the opinion of this author that the viability of this process shows promise and ought to be further considered.

\subsection{Dynamical results in comparison to other studies}

The linear instability discovered by Papaloizou \& Pringle (1984, 1985) was analytically demonstrated in an analysis of homentropic gas in slender accretion tori (i.e. not exactly rotationally supported) constrained by pressure conditions on the boundaries of the domain. This means to say that the vertical and radial scales are much smaller than the azimuthal scales and the flow is contained in a narrow annular channel. Furthermore, the modal disturbances have very nearly uniform potential vorticity throughout and have the character of being essentially vertically hydrostatic as illucidated by Goldreich et al. (1986). This instability occurs for sinuous modes (i.e. having even symmetry with respect to the disk midplane) while no such instability is observed for varicose modes (i.e. having odd with respect to the disk midplane). The instability has been physically rationalized by understanding that the pressure boundary conditions applied at the inner and outer walls of the undulating tori essentially set up a situation in which edgewaves counterpropagate with respect to each other and interact across a region which is evanescent leading ultimately to instability (Goldreich et al. 1986; Blaes \& Glatzel 1986). In view of this mechanistic understanding, the PPI should be considered the disk analog of Eady edgewaves encountered frequently in terrestrial atmospheric studies (e.g. Davies \& Bishop 1994).

The dynamics considered here are for rotationally supported disks $(q=3 / 2)$ which differ mathematically from tori $(q \neq 3 / 2)$ which have significant radial pressure support at the lowest order of the dynamics. The instability of uniform potential vorticity modes discussed in Sect. 3 and which is an incarnation of the strato-rotational instability (SRI, Dubrulle 2004) is not the same as the Papaloizou-Pringle instability (PPI). The main differences are (i) the symmetry of the modes discussed in this work are varicose while they are sinuous for the PPI; and (ii) the PPI arises in part from the pressure boundary conditions at the inner and outer boundaries while the instability here arises from the no normal flow condition at the inner and outer boundaries. Both instabilities, however, share (i) the characteristic as arising from the interaction of counterpropagating edgewaves of some sort and are explained in terms of the Hayashi-Young criterion for wave instability in shear flow (Hayashi \& Young 1987); and (ii) the character of belonging to a general class of barotropic/baroclinic instabilities related to the dynamics of potential vorticity disturbances (Bretherton 1966; Hoskins et al. 1985; Davies \& Bishop 1994).

\subsection{Future directions}

A natural test and evaluation of the shallow water theory developed in this study will be to study the nonlinear solutions of nonuniform potential vorticity disturbances. At the outset such an investigation will surely require numerical computational simulations. The question of what boundary conditions to use will be a sensitive one since, for instance, certain conditions may lead to strong linear instabilities and the relevance of them to real disks are questionable (although certainly not yet settled). For example, by requiring no-normal flow boundary conditions can excite disturbances which include uniform potential vorticity perturbations which have been shown to be the analog of the SRI. However, in light of the arguments presented here, it is possible that such boundary conditions and their resulting dynamical response could be qualitatively representative of what goes on in bonafide real disk systems.

It should also be considered that the shallow water theory developed here (by its construction) is unable to follow the dynamical response of disturbances in which there is a vertical variation in the entropy on account of the assumptions made about the thermodynamical response of the fluid made at the outset (i.e. the assumption of a polytropic gas). Vertical variations of this sort may be playing a critical role in the development of the dynamics reported in Barranco \& Marcus (2005) since it was observed in that work that vertically propagating disturbances contribute to the complex vortex dynamics seen to manifest in their simulations (see also the discussion on the stability of tall columnar vortices in Dritschel \& Juarez 1996). As one of the long term goals we have in mind for developing a shallow water formalism of non-axisymmetric disturbances is to better understand the emergence of such interesting dynamics, it will be worthwhile to extend the theory developed here in order to accommodate such circumstances by allowing for vertical variations of some type beyond the fluctuations of the disk height we follow here. One way would be to develop a multi-stacked shallow water theory in a manner similar to what has been developed in the study of MHD disturbances in the solar tachocline (Gilman 2000; Cho et al. 2007).

We would like to make one final observation pointed out by the referee of this article. With respect to the problem of stellar tachoclines Zaqarashvili et al. (2007) discuss how the inclusion of a background azimuthal magnetic field can split an ordinary Rossby wave into two different parallel modes. By analogy a similar phenomenon is likely to occur in magnetized disks (see also Matsumoto \& Tajima 1995). A strategy like the one employed in this work may be used to further detail the scope and shape of the modification of Rossby waves in such circumstances.

Acknowledgements. The author offers thanks to the anonymous referee who offered numerous suggestions to improve the quality of the presentation of this work. The author also thanks James Cho for a number of enlightening discussions. This research was supported in part by BSF grant 2004087 and ISF grant $1084 / 06$.

\section{References}

Balmforth, N. J., \& Spiegel, E. A. 1996, Phys. D, 97, 1

Balmforth, N. J., Meacham, S. Pl, Spiegel, E. A., \& Young, W. R. 1992, in Astrophysical Disks (New York Acad. Sci.), ed. J. Hunter, R. Wilson, 53 Balmforth, N. J., Howard, L. N., \& Spiegel, E. A. 1993, MNRAS, 260, 253 Balmforth, N. J., Howard, L. N., \& Spiegel, E. A. 1995, SIAM J. Appl. Math., 55,298

Barranco, J. A., Marcus, P., \& Umurhan, O. M. 2000, in Center for Turbulence Research - Proceedings of the 2000 Summer Program, 85

Barranco, J., \& Marcus, P. 2005, ApJ, 623, 1157

Blaes, O. M., \& Glatzel, W. 1986, MNRAS, 220, 253

Bodo, G., Tevzadze, A., Chagelishvili, G., et al. 2007, A\&A, 475, 51

Bretherton, F. P. 1965, Quart. J. R. Meteor. Soc., 92, 335

Bracco, A., Chavanis, P., Provenzale, A., \& Spiegel, E. A. 1999, Phys. Fluids, 11,2280 
Chagelishvili, G. D., Zahn, J.-P., Tevzadze, A. G., \& Lominadze, J. G. 2003, A\&A, 402, 401

Charney, J. G., \& Stern, M. E. 1962, J. Atmos. Sci., 19, 159

Cho, J. Y.-K., Menou, K., Hansen, B. M. S., \& Seager, S. 2007, in press

Davies, H. C., \& Bishop, C. H. 1994, J. Atm. Sci., 51, 1930

Dritschel, D. G., \& M. Torre Juarez 1996, J. Fluid Mech., 328, 129

Dubrulle, B., Marie, L. , Normand, Ch., et al. 2004, A\&A, 429, 1

Gilman, P. A. 2000, ApJ, 544, L79

Godon, P., \& Livio, M. 1999, ApJ, 523, 350

Goldreich, P., \& Lynden-Bell, D. 1965, MNRAS, 130, 125

Goldreich, P., Goodman, J., \& Narayan, R. 1986, MNRAS, 221, 339

Hayashi, Y.-Y., \& Young, W. R. 1987, J. Fluid Mech., 184, 477

Hoskins, B. J., McIntyre, M. E., \& Robertson, A. W. 1985, Quart. J. R. Meteor. Soc., 111, 877

Knobloch, E. 1984, Geophys. Astrophys. Fluid Dyn., 29, 105

Korycansky, D. G. 1992, ApJ, 399, 176

Langton, J., \& Laughlin, G. 2008, ApJ, 674, 1106

Lesur, G., \& Longaretti, P.-Y. 2005, A\&A, 444, 25

Li, H., Finn, J. M., Lovelace, R. V. E., \& Colgate, S. A. 2000, ApJ, 533, 1023

Lithwick, Y. 2007a, ApJ, 670, 789

Lithwick, Y. 2007b [arXiv: 0710.3868]

Lovelace, R. V. E., Li, H., Colgate, S. A., \& Nelson, A. F. 1999, ApJ, 513, 805
Matsumoto, R., \& Tajima, T. 1995, ApJ, 445, 767

Molemaker, M. J., McWilliams, J. C., \& Yavneh, I. 2001, Phys. Rev. Lett., 86, 5270

Papaloizou, J. C. B., \& Pringle, J. E. 1984, MNRAS, 208, 721

Pedlosky, J. 1987, Geophysical Fluid Dynamics, 2nd edn (New York: SpringerVerlag)

Petersen, M. R., Julien, K., \& Stewart, G. R. 2007a, ApJ, 658, 1236

Petersen, M. R., Stewart, G. R., \& Julien, K. 2007b, ApJ, 658, 1252

Salby, M. L. 1989, Tellus, 41A, 48

Schmid, P. J., \& Henningson, D. S. 2001, Stability and Transition in Shear Flows (Springer)

Showman, A. P., Menou, K., \& Cho, J. Y-K. 2007

[arXiv: 0710.2930v1]

Sternberg, A. 2005, A search for Hydrodynamical Instabilities in Accretion Disks, Masters Research Thesis, Technion - Israel Insitute of Technology

Tevzadze, A. G., Chagelishvili, G. D., Zahn, J.-P., Chanishvili, R. G., \& Lominadze, J. G. 2003, A\&A, 407, 779

Tevzadze, A. G., Chagelishvili, G. D., \& Zahn, J.-P. 2008, A\&A, 478, 9

Umurhan, O. M. 2006, MNRAS, 365, 85

Umurhan, O. M., \& Regev, O. 2004, A\&A, 427, 855

Zaqarashvili, T. V., Oliver, R., Ballester, J. L., \& Shergelashvili, B. M. 2007, A\&A, 470,815 